\title{
Circadian Clock Gene Plays a Key Role on Ovarian Cycle and Spontaneous Abortion
}

\author{
Ruiwen Liacc Shuting Cheng ${ }^{a, b} \quad$ Zhengrong Wang ${ }^{a, b}$ \\ aMinistry of Health Key Laboratory of Chronobiology, College of Basic Medicine and Forensic Medicine, \\ Sichuan University, Chengdu, Sichuan, 'bState Key Laboratory of Biotherapy, Sichuan University, \\ Chengdu, Sichuan, 'Reproductive Laboratory, Chengdu Woman Children Central Hospital, Chengdu, \\ Sichuan, P. R. China
}

\section{Key Words}

CLOCK • Female fertility - Spontaneous abortion - Ovarian cycle $\cdot$ Induced abortion $•$ Clock gene knockout

\begin{abstract}
Background/Aims: Circadian locomotor output cycles protein kaput (CLOCK) plays a key role in maintaining circadian rhythms and activation of downstream elements. However, its function on human female reproductive system remains unknown. Methods: To investigate the potential role of CLOCK, CLOCK-shRNAs were transfected into mouse 129 ES cells or injected into the ovaries of adult female mice. Western blotting was utilized to analyze the protein interactions and flow cytometry was used to assess apoptosis. Results: The expression of CLOCK peaked at the 6th week in the healthy fetuses. However, an abnormal expression of CLOCK was detected in fetuses from spontaneous miscarriage. To determine the effect of CLOCK on female fertility, a small hairpin RNA (shRNA) strategy was used to specifically knockdown the CLOCK gene expression in vitro and in vivo. Knockdown of CLOCK induced apoptosis in mouse embryonic stem (mES) cells and inhibited the proliferation in mES cells in vitro. CLOCK knockdown also led to decreased release of oocytes and smaller litter size compared with control in vivo. Conclusions: Collectively, theses findings indicate that CLOCK plays an important role in fertility and that the CLOCK knockdown leads to reduction in reproduction and increased miscarriage risk.
\end{abstract}

Copyright (C) 2015 S. Karger AG, Basel

\section{Introduction}

The number of miscarriage is increasing among women in different age groups, especially in aged and high-risk groups. Circadian locomotor output cycles protein kaput (CLOCK), a key regulator of the circadian rhythm, was found to be related to miscarriage and low number of pregnancies [1]. CLOCK is a transcription factor of the basic helix-loop- 
helix (bHLH) family encoded by the circadian locomotor that plays a central role in the circadian system [2] . Circadian rhythms are regulated by the suprachiasmatic nuclei (SCN) of the anterior hypothalamus [3], which is the "master clock" of the mammal brain [4]. The circadian CLOCK is located within SCN, coordinating peripheral oscillators [5] that maintain the timing for a range of physiological functions including endocrine, cardiac functions, body temperature and physical activities. Mutant CLOCK gene shows disrupted estrous cycle and increased rates of full-term pregnancy failure [6]. Our previous study demonstrated that knockdown of the CLOCK on mice led to smaller litter size, lower fertility rate, lower blastula formation rate and lower acrosin activity. Collectively, the gene expression of CLOCK in round spermatid is essential for maintaining male reproductivity in vivo [7].

CLOCK has protein dimerization activity and sequence-specific DNA binding transcription factor activity regulated by ARNTL2 paralog [8]. In human, ARNTL2-CLOCK activates E-box element (5'-CACGTG-3') of the circadian clock. Transcriptions of PER1 and PER2 are inhibited in a feedback loop with CLOCK/ARNTL complexes of PER and CRY proteins. It contributes to chromatin remodeling events, which implicates the circadian control of gene expression.

Embryonic stem (ES) cells are derived from an embryonic blastocyst and featured with the prolonged asymmetrical division with the potential to differentiate into many cell types [9]. Because of its pluripotency and self-renewing property, ES cells can be used to study differentiation in vitro.

This study adopted the small hairpin RNA strategy to specifically knockdown Clock in mouse ES (mES) cells and to knock out Clock in the ovaries of female mice to estimate the effects on the cell differentiation and fertility rate. The CLOCK expression levels were examined in the samples from women who had spontaneous miscarries or induced abortion to explore the underlying mechanisms of CLOCK. Hereby, we aimed to determine the effects of CLOCK and knockdown of CLOCK on female fertility.

\section{Materials and Methods}

\section{Selection of Patients}

This study was approved by the Ethical Committee of Chengdu Woman Children Central Hospital and formal consents were obtained from each patient. All fetus tissues including induced abortion and spontaneous miscarriage in this study were provided from the Department of Obstetrics and Gynecology, Chengdu Woman Children Central Hospital of China. A total of 58 spontaneous miscarriage fetuses and 40 induced abortion fetuses were obtained at gestational age ranged from 5 to 9 weeks, which was established by menstrual history and confirmed by ultrasonography. The specimen from induced abortion fetus was collected by vaginal curettage. In spontaneous miscarriage, curettage was performed within $24 \mathrm{~h}$ after diagnosis. None of the abortion was pharmacologically induced. Both groups were screened in same exclusionary criteria. Women with any medication, genetic defects, cases of chromosomal, hormonal or uterine structural abnormalities, and cases associated with infectious, autoimmune, or other systemic or local diseases were excluded. Specimen was immediately frozen in liquid nitrogen for following experiments.

Mouse ES cells culture

Mouse 129 ES Cells were obtained from the American Type Culture Collection (Manassas, VA). The mES cells were cultured in Dulbecco's Modified Eagle's Media (DMEM, Gibco, USA) supplemented with 3.7 g/L sodium bicarbonate, $1 \%$ penicillin and streptomycine, $1.7 \mathrm{mM}$ L-glutamine, $0.1 \mathrm{mM}$ beta mercaptoethanol, $5 \mathrm{ng} / \mathrm{mL}$ mouse leukemia inhibitory factor (LIF), and 15\% FBS.

CLOCK ShRNA lentivirus production and infection

To construct CLOCK shRNA vectors, 2 pairs of independent oligo primers targeting CLOCK was subcloned into PLKO.1 vector (Addgene). Scramble shRNA vectors was applied as control. The sequences of CLOCK or scamble shRNA were as follows: Scramble shRNA (Scr): 5'-GGA ACA TCA GGC TAT GAT TCT-3'; CLOCK shRNA1 (sh-1): 5'-GTG CTT CAG ATG TCC ATT AAA-3'; CLOCK shRNA2 (sh-2): 5'-CGG ATG ATA GAG 


\section{Cellular Physiology Cell Physiol Biochem 2015;37:911-920 \\ \begin{tabular}{l|l|l}
\hline DOI: 10.1159/000430218 & (C) 2015 S. Karger AG, Basel
\end{tabular} \\ and Biochemistry Published online: September 18, $2015 \quad$ www.karger.com/cpb \\ Li/Cheng/Wang: CLOCK Gene in Spontaneous Abortion}

GCA AAT ATT-3'. Then lentivirus production was performed in HEK 293T cells. For infection, feeder-free ES cells were plated at a density of $3 \times 10^{5}$ cells/well in 6-well plate. After $24 \mathrm{~h}$, lentiviruses of CLOCK shRNA or scramble shRNA were added to cells in fresh medium containing $8 \mu \mathrm{g} / \mathrm{mL}$ polybrene. After $48 \mathrm{~h}$, knockdown efficiencies were detected by quantitative real-time RT-PCR (qRT-PCR) or Western blot analysis.

\section{Quantitative Real-time PCR}

The mRNA expression of CLOCK and $\beta$-actin during the different stages of induced abortion fetuses (5 to 9th week) was quantified by real-time PCR. Specific PCR primers were designed according to the sequences of target genes of Homo sapiens. The amplification mixture contained $1 \mu \mathrm{L}$ of RT reaction mix, $10 \mu \mathrm{L}$ of SYBR ${ }^{\oplus}$ Premix Ex Taq ${ }^{\text {TM }}(2 \times)$ (TaKaRa, China), $0.5 \mu \mathrm{L}$ of $10 \mu \mathrm{mol} / \mathrm{L}$ each of primers and $8.5 \mu \mathrm{L}$ $\mathrm{ddH}_{2} \mathrm{O}$. Reactions were performed on a fluorescence temperature cycler (Bio-Rad, Hercules, CA, USA). The PCR conditions for CLOCK and $\beta$-actin were as follows: one cycle of 4 min at $95^{\circ} \mathrm{C} ; 40$ cycles of $30 \mathrm{~s}$ at $95^{\circ} \mathrm{C}$, $30 \mathrm{~s}$ at $63^{\circ} \mathrm{C} / 57^{\circ} \mathrm{C}, 30 \mathrm{~s}$ at $72^{\circ} \mathrm{C}$. The sequences of CLOCK $/ \beta$-actin primers in PCR are listed as follows:

CLOCK, Forward: 5'-ACA GGG CAC CAC CCA TAA TAG-3', Reverse: 5'-AAT GAG TCT GAA GCC AAA TCC A-3'; $\beta$-actin, Forward: 5'- CCA CAC TGT GCC CA TCT ACG-3', Reverse: 5'-ATG TCA CGC ACG ATT TCC C-3'. The threshold cycle (CT) resulting from RT-PCR was analyzed using the $2^{-\Delta \Delta C t}$ method.

\section{Western blot}

Cells were harvested, washed twice with PBS, and lysed in lysis buffer (20 mM Tris [pH 7.5], $1 \mathrm{mM}$ EDTA, $1 \mathrm{mM}$ EGTA, 1\% Triton X-100, $1 \mathrm{mg} / \mathrm{ml}$ aprotinin, $1 \mathrm{mM}$ phenylmethylsulfonylfluoride [PMSF], and $0.5 \mathrm{mM}$ sodium orthovanadate) for $30 \mathrm{~min}$ on ice. The lysates were cleared by centrifugation (30 min at $12,000 \mathrm{~g}, 4^{\circ} \mathrm{C}$ ), and the protein concentration was determined using the Bradford method. Briefly, equal amounts of protein were resolved by $10 \%$ SDS-PAGE and transferred to polyvinylidenedifluoride (PVDF) membranes. The membranes were washed with TBST (10 mM Tris- $\mathrm{HCl}$ [pH 7.6], $150 \mathrm{mM} \mathrm{NaCl}$, and $0.01 \%$ Tween-20), blocked with TBST containing $5 \%$ skim milk for $1 \mathrm{~h}$, and incubated with the primary antibody against CLOCK, Bcl-2, Bax, NFKB-p65 and Cleaved Caspase-3 (1:1000 dilution, Sigma-Aldrich, USA) and the primary antibodies were detected with goat anti-rabbit IgG conjugated to horseradish peroxidase $(1: 5000$ dilution, Sigma-Aldrich, USA). Immunoreactive proteins were visualized by enhanced chemiluminescence (Amersham Pharmacia Biotech, Buckinghamshire, UK).

\section{Immunohistochemical staining}

The formaldehyde-fixed, paraffin-embedded chorionic villi tissues were cut into 4-5 $\mu \mathrm{m}$ sections, using mouse anti-human CLOCK antibodies (1:200, R\&D Systems, Inc., USA) for the immunohistochemical staining. The sections were deparaffinized, rehydrated and washed with PBS, then immersed in 3\% hydrogen peroxide to block endogenous peroxidase and antigens were repaired with a pressure cooker. The sections were incubated for $30 \mathrm{~min}$ at $37^{\circ} \mathrm{C}$ before incubated with the primary antibodies overnight at $4^{\circ} \mathrm{C}$. The Sections were incubated at room temperature with the secondary antibodies for $1 \mathrm{~h}$. The sections were then incubated in DAB exposure medium. DAB exposure medium was washed off and the sections were stained with hematoxylin for $30 \mathrm{~s}$. The Positive expression was indicated by brown-yellow staining in five discontinuous visual fields under high magnification $(\times 400)$. The images were analyzed using ImagePro Plus 6.0 software (Media Cybernetics, Rockville, MD). The CLOCK expression intensities in the chorionic villi were analyzed in terms of integral optical density (IOD).

\section{MTT}

Cell viability was determined using the conversion of MTT to formazan via mitochondrial oxidation. mES cells were pretreated with different shRNAs and placed in 96-well plates (Corning Inc., Corning, New York, USA) at $1 \times 10^{3}$ cells/well and then cultured at 4 different times $(24,48,72$, and $92 \mathrm{~h})$, At the indicated time, MTT solution was added to each well at a final concentration of $1 \mathrm{mg} / \mathrm{mL}$ per well and the plates were incubated for another $2 \mathrm{~h}$. After incubation, $150 \mu \mathrm{L}$ of DMSO was added to each well to dissolve the formazan formed for $10 \mathrm{~min}$ and the absorbance was read at $540 \mathrm{~nm}$ using a Synergy HT microplate reader (BioTek Instruments, Inc., Winooski, VT, USA).

Annexin Vassay

FITC Annexin V Apoptosis Detection Kit II (Pharmingen, USA) was used to analyze the apoptosis by flow cytometry. After treatment with different infection for $48 \mathrm{~h}$, the mES cells were collected and washed 
twice with cold PBS. The resuspended cells in $100 \mu \mathrm{l}$ of $1 \times$ binding buffer were transferred to a $5 \mathrm{ml}$ culture tube, and $5 \mu$ of FITC Annexin V was added. After gently vortexing, the cells were incubated for $15 \mathrm{~min}$ at room temperature in the dark. Finally, $400 \mu \mathrm{l}$ of the $1 \times$ binding buffer were added to each tube, and analyzed by flow cytometry.

\section{Plasmid transfection and breeding}

The scramble shRNA (Scr) or CLOCK shRNA1 (sh-1) plasmids were injected into the ovaries of adult female mice ( $\mathrm{n}=20$ each group) by an in vivo jetPEI (Polyplus, IllkirchCedex, France), and 18 days post injection, these females were bred with normal adult male mice.

\section{Determination of spontaneous oocyte release}

Vaginal smears were monitored for 2 estrous cycles until a proestrus smear. During the estrous stage, mice were sacrificed and the ovaries with attached oviducts were removed. The ovary and oviduct were further dissected in Hank's balanced salt solution (HBSS; Gibco, Invitrogen, Carlsbad, CA) to remove the fat and uterus and were transferred to fresh solution in an indented microscopic slide for analysis. Each oviduct was carefully examined for released oocytes by making a small incision in the oviduct and teasing out the contents of the entire oviduct. The cumulus mass was dissociated by treatment in HBSS containing $300 \mu \mathrm{g} /$ $\mathrm{mL}$ hyaluronidase (Sigma-Aldrich, St Louis, MO), and the oocytes were distinguished from other detritus by their spherical nature.

\section{Measurement of Fertility}

Female mice at 2 months old were paired with male WT mice ( 2 months of age), then the number of litter size were counted to determine fertility.

\section{Statistical analysis}

All data are expressed as mean \pm SD. The statistical significance of differences between experimental groups was determined using one-way ANOVA by Student's t-test (SPSS 11.0, SPSS Inc., Cary, NC). Probability values $(P)$ of less than 0.05 were considered statistically significant.

\section{Results}

CLOCK expression peaks at the 6th week in the induced aborted fetuses

The mRNA and protein expression of CLOCK from human induced aborted fetuses from week 5 to week $9(n=40)$ were obtained and analyzed. Both mRNA and protein expression of CLOCK reached the peak level at week $6(P<0.01)$ and declined in week 7 to 9 but higher than the week 5 ( $P<0.05$ vs. week 5) (Fig. 1A and B).

The lower mRNA and protein of CLOCK in spontaneous aborted fetuses than in induced aborted fetuses induces cell apoptosis

Among the spontaneous aborted (miscarriages) fetuses $(n=58)$, both mRNA and protein expressions of CLOCK were more than $50 \%$ lower than those in induced aborted $(\mathrm{n}=40$ ) (induced aborted fetuses $74 \%$ vs. spontaneous aborted fetuses 32\%) (Fig. 1C and D). The mRNA and protein expression levels of CLOCK were analyzed by qRT-PCR and Western blotting, respectively. The immunohistochemical staining of CLOCK in the fetuses from induced abortion or spontaneous abortion revealed a lower CLOCK expression in the chorionic villi of spontaneous abortion fetuses (Fig. 1E). The results indicated that the expression intensities of CLOCK in integral optical density (IOD) were lower in miscarriage fetuses than in the induced aborted group (IOD: SA $34.0 \pm 4.5$ versus $72.0 \pm 5.0, P<0.01$, Fig. 1F). Moreover, the cleaved caspase- 3 expression was significantly higher in spontaneous aborted fetuses than in induced aborted fetuses $(P<0.05$, Fig. $1 \mathrm{G})$. Thus, the lower expression of CLOCK could be the cause to induce cell apoptosis, thus introducing the spontaneous abortion. 


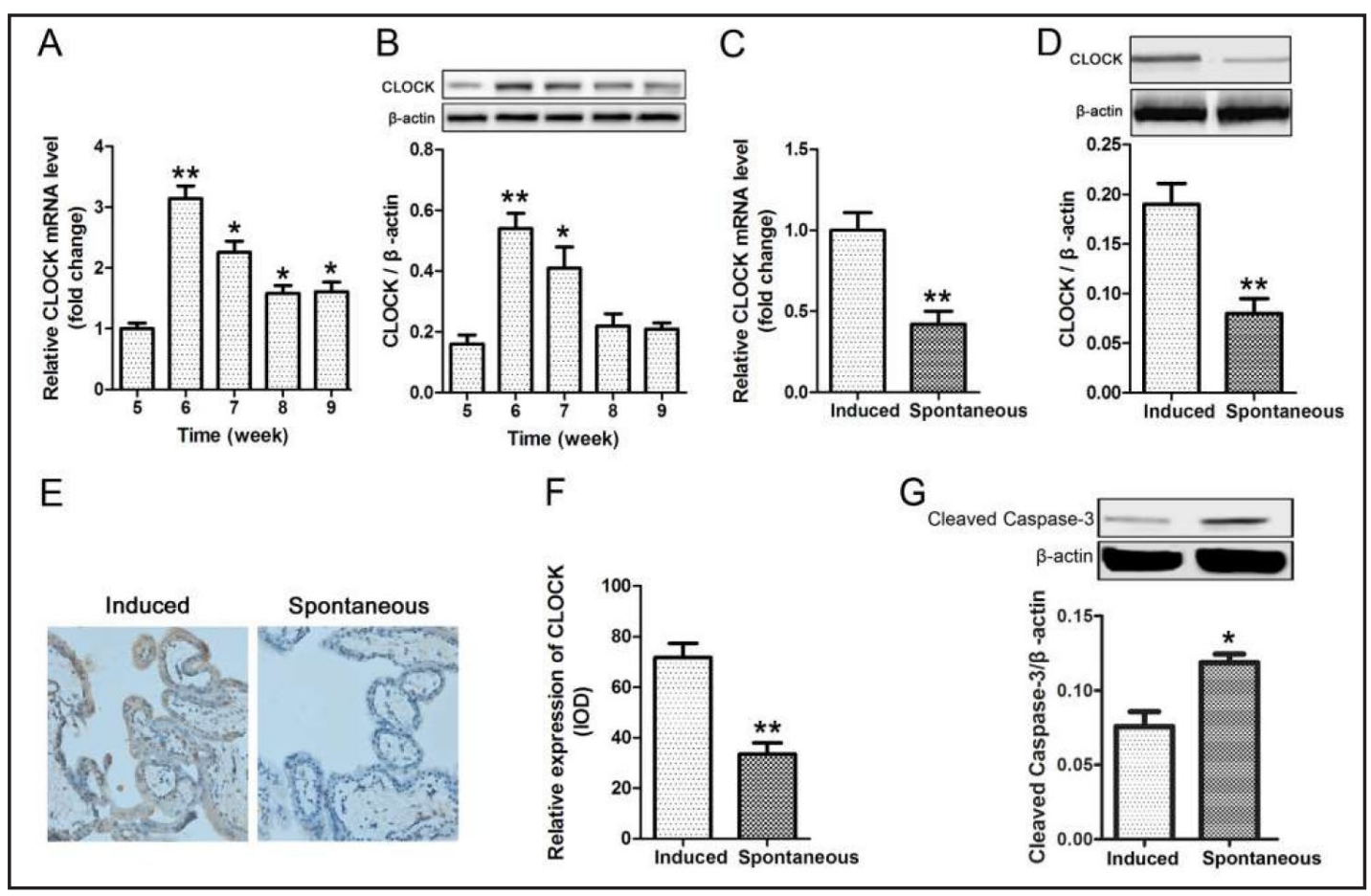

Fig. 1. The difference expression of CLOCK gene between in normal human induced abortion and spontaneous miscarriage. (A-B) CLOCK mRNA (A) and protein (B) expression levels in normal human induced abortion fetuses in various weeks were analyzed by qRT-PCR and Western blotting with $\beta$-actin as the loading control, respectively. The mRNA expression levels were normalized to $\beta$-actin. The Western blotting results were normalized to $\beta$-actin. The data represent means \pm SD of 3 independent experiments. * and ** denotes $P<0.05$ and $P<0.01$ compared with week 5. (C-G) The expression of CLOCK mRNA (C) and protein (D) in the fetuses of normal induced abortion and spontaneous miscarriage were analyzed by qRT-PCR and Western blotting with $\beta$-actin as the loading control, respectively. The mRNA expression levels were normalized to $\beta$-actin. (E) Immunochemical analysis (IHC) of CLOCK in the fetuses of normal induced abortion and spontaneous miscarriage. (F) The CLOCK expression intensities were analyzed in terms of integral optical density (IOD). (G) The expression of cleaved caspase-3 in the fetuses of normal induced abortion and spontaneous miscarriage were analyzed by Western blotting with $\beta$-actin as the loading control. The data represent means \pm SD of 3 independent experiments. * and ${ }^{* *}$ denotes $P<0.05$ and $P<0.01$ compared with the induced aborted group.

CLOCK mRNA and protein expressions were reduced by CLOCK-shRNAs

To assess the effects of CLOCK, all shRNAs (Scr, sh-1 and sh-2) were transduced into the mES cells to knockdown CLOCK. mES cells without lentivirus plasmid were used as control. As shown in Fig. 2A and B, $48 \mathrm{~h}$ after transduction, both sh- 1 and sh- 2 groups showed significantly downregulated mRNA and protein levels of CLOCK compared with the control groups $(P<0.01)$. Compared with the control group, there was a significant inhibition in cell growth in sh-1 and sh-2 groups (Fig. 2C). Moreover, there were also obvious higher apoptosis rates in sh-1 $(25.08 \%)$ and sh-2 group $(20.66 \%)$ than control $(5.42 \%)$ groups $(P<0.05)$ (Fig. $2 \mathrm{D})$. The results suggested that the knockdown of CLOCK increase the apoptosis rate of cells, thus leading to cell death.

CLOCK-shRNA inhibits the expression of Bcl-2 and upregulates the expression of bax, cleaved caspase-3 and $N F \kappa B-p 65$

To further determine the impact of CLOCK on apoptosis, the expressions of apoptosis related proteins Bcl-2, bax, cleaved caspase-3 and NFKB-p65 expression, which is involved in the apoptotic signal pathway, were examined in these different treated groups. Results demonstrated that Bcl- 2 activity was significantly inhibited in sh- 1 and sh-2 groups compared 


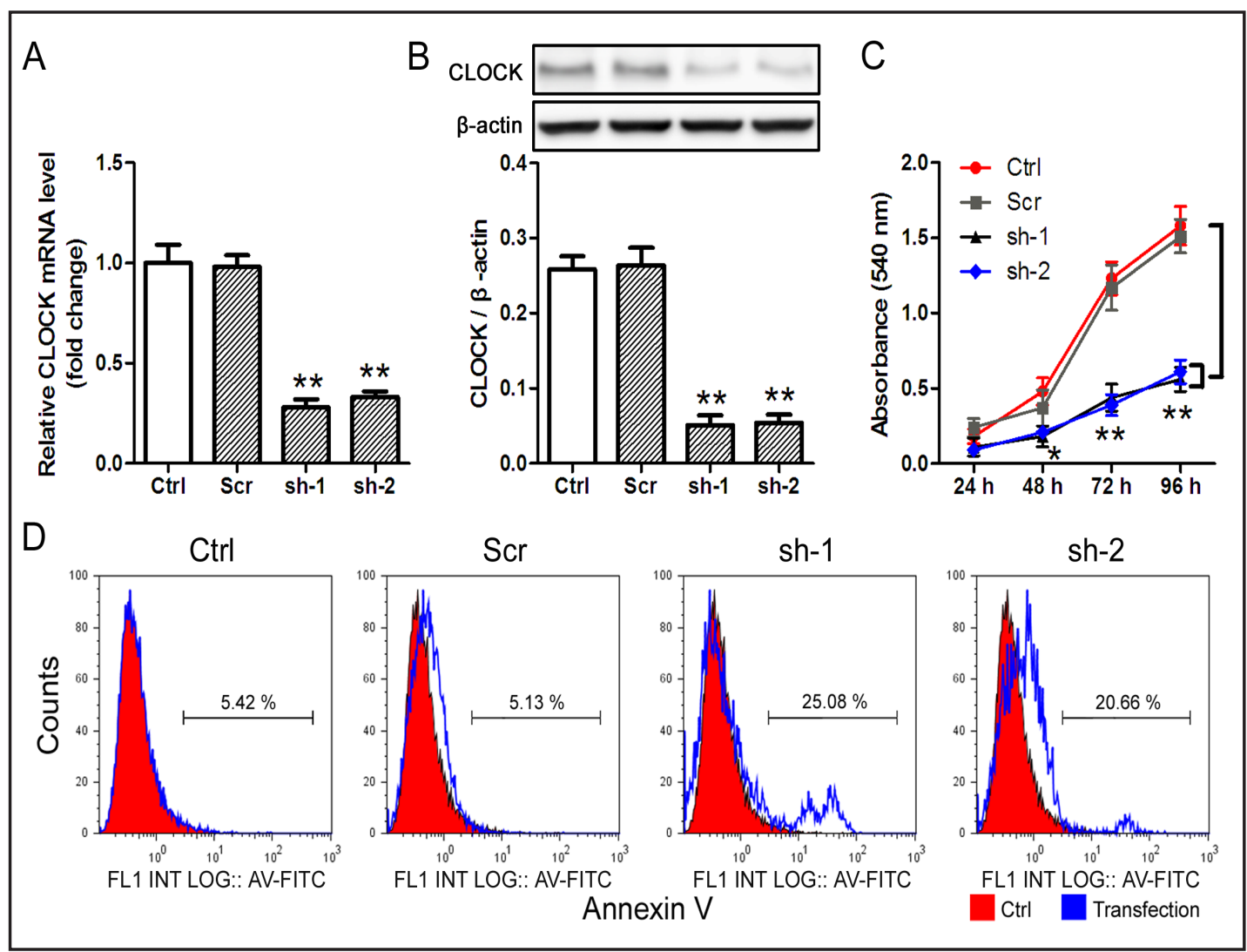

Fig. 2. The expression and apoptosis was analyzed in mES cells with knockdown of CLOCK. The mouse embryonic stem (mES) cells were infected with lentiviruses of scramble shRNA (Scr), CLOCK shRNA1 (sh-1), or CLOCK shRNA2 (sh-2). The cells with no infection were as normal control (Ctrl). (A-B) The expression of CLOCK mRNA (A) and protein (B) in the mES cells with different infections for $48 \mathrm{~h}$ were analyzed by qRT-PCR and Western blotting with $\beta$-actin as the loading control, respectively. The mRNA expression levels were normalized with $\beta$-actin. The data represent means \pm SD of 3 independent experiments. ${ }^{* *} P<0.01$ compared with the Ctrl group. (C) Mean growth rate of the mES cells measured by MTT assay. The data represent means \pm SD of 3 independent experiments. ${ }^{*} P<0.05,{ }^{* *} P<0.01$ compared with the Ctrl group. (D) The apoptotic cell death of the mES cells with different infections for $48 \mathrm{~h}$ was analyzed using the annexin V assay.

with control group $(P<0.01$, Fig. 3A). In contrast, bax, cleaved caspase-3 and NFkB-p65 were upregulated in both sh-1 and sh-2 groups compared with the control group $(P<0.01$, Fig. 3B, C and D).

PDTC can inhibit the upregulation of NFKB-p65 expression induced by CLOCK-shRNA

In this study, to test whether CLOCK can induce NF- $\kappa B$ signal pathway, pyrrolidine dithiocarbamate (PDTC), a selective nuclear factor- $\kappa \mathrm{B}$ (NF- $\kappa \mathrm{B}$ ) inhibitor, were treated to the cells for $1 \mathrm{~h}$. As showed in Fig. 3E and F, sh-1 transduction caused the silence of CLOCK and introduced the upregulation of NFKB-p65 in mES cells. The PDTC treatment can reverse the CLOCK knockdown-induced upregulation of NFKB-p65 $(P<0.01)$, but did not affect the expression of CLOCK (Fig. 3E and F). Moreover, the apoptotic rates were compared among different treated groups. CLOCK shRNA-transfected group had higher apoptosis $(21.51 \%)$ compared with the control group (3.18\%) $(P<0.05)$ and PDTC without CLOCK-shRNA group. However, PDTC treatment can reduce the high apoptosis rate caused by CLOCK knockout (10.13\%) (Fig. 3G). 


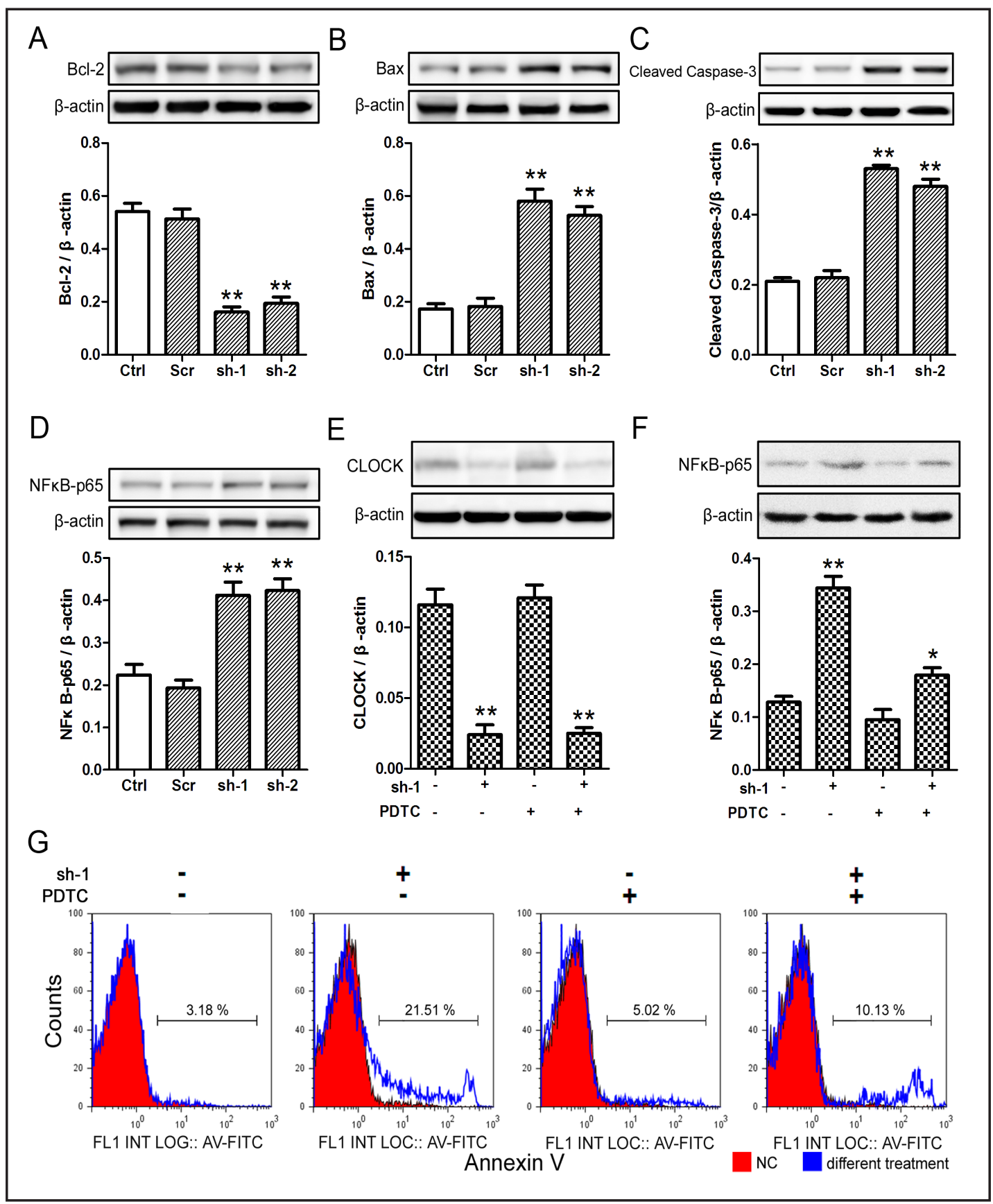

Fig. 3. CLOCK-shRNA inhibites the expression of Bcl-2 and Up-regulates the Bax and NFKB-p65 expression, but PDTC can reverse the upregulation of NFKB-p65 expression induced by CLOCK-shRNA. (A-D) The mES cells were infected with lentiviruses of scramble shRNA (Scr), CLOCK shRNA1 (sh-1), or CLOCK shRNA2 (sh2) for $48 \mathrm{~h}$. The cells with no infection cultured for $48 \mathrm{~h}$ were as normal control (Ctrl). Protein expression levels of Bcl-2 (A), Bax (B), Cleaved Caspase-3 (C) and NFкB-p65 (D) were analyzed by Western blotting with $\beta$-actin as the loading control. The data represent means \pm SD of 3 independent experiments. ${ }^{* *} P<0.01$ compared with the Ctrl group. (E-G) The mES cells were infected with lentiviruses of CLOCK shRNA1 (sh1), or pretreated with Pyrrolidine dithiocarbamate (PDTC), a selective nuclear factor- $\kappa B(N F-\kappa B$ ) inhibitor for $1 \mathrm{~h}$. Protein expression levels of CLOCK (E) and NFKB-p65 (F) were analyzed by Western blotting with $\beta$-actin as the loading control. (G) The apoptotic cell death was analyzed using the annexin $V$ assay. The data represent means \pm SD of 3 independent experiments. ${ }^{*} P<0.05,{ }^{* *} P<0.01$ compared with the untreated group. 


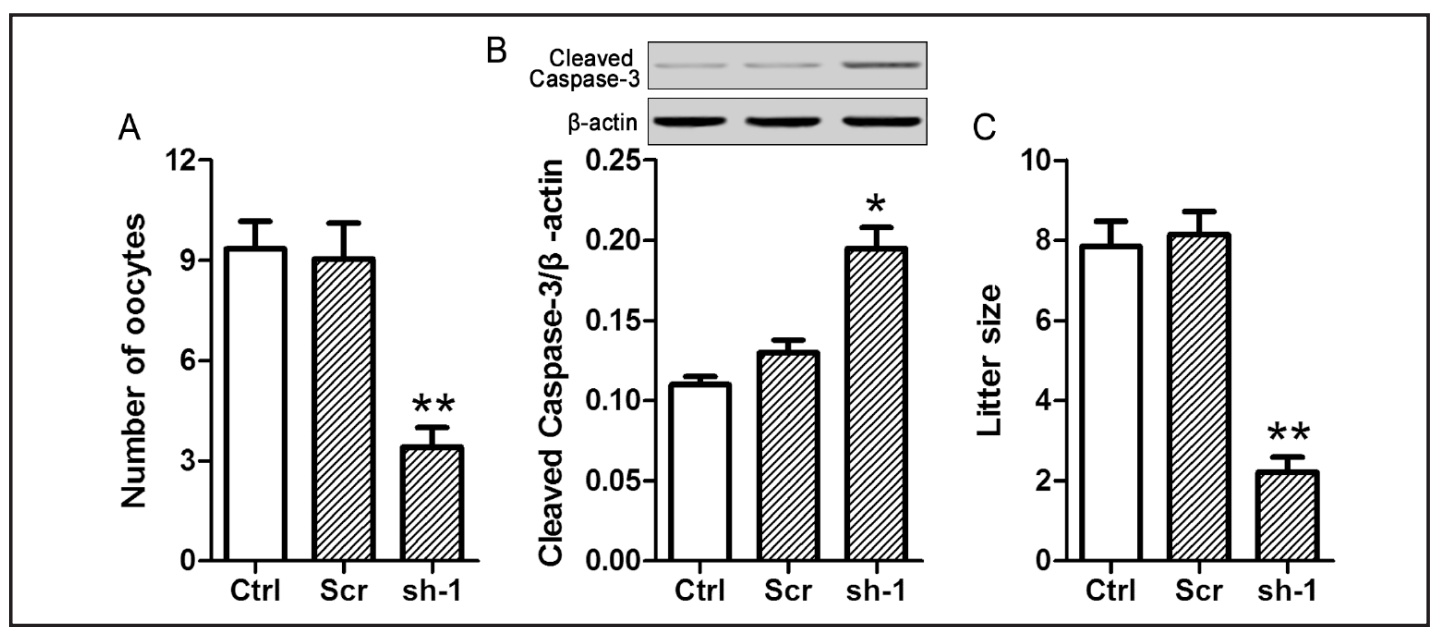

Fig. 4. Knockdown of CLOCK gene affects on mice fertility. The scramble shRNA (Scr) or CLOCK shRNA1 (sh-1) plasmids were transfected into the ovaries of adult female mice ( $\mathrm{n}=20$ each group), and 18 days post injection these females were bred with normal adult male mice. Mice without any treatment served as normal control (Ctrl). (A) The number of oocytes spontaneously released on the morning of the estrous stage. (B) The expression of Cleaved Caspase-3 in oocytes was analyzed by Western blotting with $\beta$-actin as the loading control. (C) The litter size was determined within 24 hours of birth. The data represent means \pm SD of 3 independent experiments. ${ }^{*} P<0.05,{ }^{* *} P<0.01$ compared with the Ctrl group.

\section{CLOCK gene knockdown reduced the ovarian cycles}

After knockdown of CLOCL by transfecting sh-1, the number of oocytes releases (Fig. 4A), the expression of cleaved caspase-3 (Fig. 4B) and the litter size (Fig. 4C) were assayed. Compared with the control group and Scr group, sh-1 showed significant reduction on the number of oocytes and litter size $(P<0.01)$ (Fig. $4 \mathrm{~A}$ and $\mathrm{C}$ ), and increase the expression of cleaved caspase-3, which indicates cell apoptosis $(P<0.05)$ (Fig. 4B).

\section{Discussion}

Reproductive ability in humans is quite complex. One third of conceptions end up in miscarriages in female, among them $10-15 \%$ are clinically recognized [10]. There are different causes to miscarriages, such as genetic, anatomical, endocrine, immune, infection, thrombophilic or unexplained reasons. Reproduction procedures are dominantly influenced by the circadian system [11, 12]. The function of CLOCK is an essential activator of downstream elements in the potential signaling pathway, which is critical to the generation of circadian rhythms [1], therefore, the circardian system gene CLOCK plays a key role in the regulation of circardian rhythms. Circadian CLOCK regulates metabolic, cell division, sleep-wake, circardian and seasonal cycles. Abnormalities such as mutations in the CLOCK genes can affect circardian activity and be hazardous to health $[13,14]$. It was reported that CLOCK mutants disrupted estrous cycles and increased rates of full-term pregnancy failure [15]. However, the impact of the loss of CLOCK on female reproduction has not been clearly assessed yet.

In the present study, the expression of CLOCK in induced aborted fetuses we examined. The results suggested that both mRNA and protein level of CLOCK reached its peak in the 6th week after pregnancy. Compared with induced aborted fetuses, the expression of CLOCK was significantly lower in the spontaneous abortion fetuses in mRNA and protein level. These results demonstrated that there was an abnormal CLOCK expression in spontaneous aborted fetuses. However, the mechanism needs to be further elucidated. Thus we hypothesize that low CLOCK expression in pregnant women may lead to abortion. 


\section{Cellular Physiology Cell Physiol Biochem 2015;37:911-920 \\ \begin{tabular}{l|l|l|}
\hline DOI: 10.1159/000430218 & O 2015 S. Karger AG, Basel
\end{tabular} \\ www.karger.com/cpb \\ Li/Cheng/Wang: CLOCK Gene in Spontaneous Abortion}

Studies have shown potential relationship between CLOCK and apoptosis, Gaddameedhi $S$ et al. reported that the DNA replication is regulated by the circadian clock. The circadian clock controls sunburn apoptosis and erythema in mouse skin [16]. Antoch MP et al. demonstrated that the upregulation of several proapoptic genes in a CLOCK mutant mouse spleen [17]. Peyric E et al. also reported that the intestinal clock controls the expression of key cell cycle regulators, such as cdc2, wee1, p21, PCNA and cdk2, but only weak influences on cyclin B1 and cyclin E1 expression [18]. These findings provide new insights into the function of the CLOCK gene in the regulation of apoptosis. It is still unclear whether the CLOCK contributes to the regulation of female fertility by controlling the apoptosis.

To verify our hypothesis, we used the small shRNA strategy to knockdown the CLOCK expression in mES cells. Results showed that knockdown of CLOCK inhibits cell growth and increases the apoptosis rate of mES cells. As a primary event in determining the susceptibility to apoptosis, the expression of apoptosis related proteins was assayed. The results indicated that anti-apoptotic protein bcl-2 was significantly decreased, and, the pro-apoptotic protein bax, cleaved caspase-3 and NFKB-p65 were significantly increased. These data demonstrated a disruption in the balance of the integrity of the mitochondria and activation of the caspase cascade [7]. These results suggest that knockdown of CLOCK induces downregulation of Bcl2 and upregulation of Bax, thus causing the apoptosis. Furthermore, cleaved caspase- 3 was upregulated in spontaneous aborted fetuses than in induced aborted fetuses. Moreover, a higher expression of cleaved caspase-3 was detected in CLOCK-shRNA group than control and Scr group. In addition, the increased level of NFKB-p65 in CLOCK-shRNA infected cells was also detected.

Previous studies indicated that the core circadian clock activity regulates the NF- $\mathrm{BB}$ pathway [19]. NF- $\kappa B$ is an essential transcription factor in many type of cellular processes, such as cellular metabolism and chemotaxis. Phosphorylation and acetylation of p65 are crucial in post transcriptional modifications required for NF- $\kappa B$ activation [20]. Therefore, the NFKB-p65 subunit is responsible for NF- $\kappa B$ heterodimer formation, nuclear translocation and activation [21]. To investigate the role of NF- $\kappa B$ cascade activation, its inhibitor, PDTC, was used together with CLOCK-shRNA treated mES cells. PDTC is a chemical compound, which can induce the G1 phase and cause cell cycle arrest [22]. It was showed that PDTC had selectivity in vivo in inhibiting NF- $\kappa B$ activation and expression of inflammatory genes [23]. In our current study, the PDTC treatment can partly prevent the upregulation of NFKB-p65 in CLOCK-shRNA infected cells. The apoptosis rate was inhibited with the treatment of PDTC in the CLOCK-shRNA infected cells. These results indicated that the knockdown of CLOCK affects the NF- $\kappa B$ signal pathway, and causes the following unbalance ratio of Bcl-2/Bax, thus inducing caspase cascade activation and apoptosis.

Moreover, the in vivo study suggested that the female mice injected with CLOCKshRNA showed lower number of oocytes, litter size and higher cell apoptosis. These results demonstrated that CLOCK has a significant role on fertility and the downregulation of CLOCK leads to cell apoptosis and reduced reproduction, thus increasing miscarriage risk.

\section{Acknowledgements}

This study was supported by the Foundation of The National Nature Science Foundation of China (No. 31371180 to ZW) and China Medical Board (CMB) (No. 88-486 to ZW).

\section{Disclosure Statement}

The authors stated that they had no interests, which might be perceived as posing a conflict. 


\section{Cellular Physiology Cell Physiol Biochem 2015;37:911-920 \begin{tabular}{l|l|l} 
DOI: 10.1159/000430218 & (C) 2015 S. Karger AG, Basel
\end{tabular} www.karger.com/cpb \\ Li/Cheng/Wang: CLOCK Gene in Spontaneous Abortion}

\section{References}

1 Dunlap JC: Molecular bases for circadian clocks. Cell 1999;96:271-290.

2 Hardin PE: From biological clock to biological rhythms. Genome Biol 2000;1:REVIEWS1023.

3 Tsuchiya Y, Minami I, Kadotani H, Nishida E: Resetting of peripheral circadian clock by prostaglandin e2. EMBO Rep 2005;6:256-261.

4 Wu YH, Fischer DF, Kalsbeek A, Garidou-Boof ML, van der Vliet J, van Heijningen C, Liu RY, Zhou JN, Swaab DF: Pineal clock gene oscillation is disturbed in alzheimer's disease, due to functional disconnection from the "Master clock". FASEB J 2006;20:1874-1876.

5 Menaker M, Murphy ZC, Sellix MT: Central control of peripheral circadian oscillators. Curr Opin Neurobiol 2013;23:741-746.

6 Miller BH, Olson SL, Turek FW, Levine JE, Horton TH, Takahashi JS: Circadian clock mutation disrupts estrous cyclicity and maintenance of pregnancy. Curr Biol 2004;14:1367-1373.

7 Liang X, Cheng S, Jiang X, He X, Wang Y, Jiang Z, Hou W, Li S, Liu Y, Wang Z: The noncircadian function of the circadian clock gene in the regulation of male fertility. J Biol Rhythms 2013;28:208-217.

8 Nakao A, Nakamura Y, Shibata S: The circadian clock functions as a potent regulator of allergic reaction. Allergy 2015;70:467-473.

9 Chambers I: The molecular basis of pluripotency in mouse embryonic stem cells. Cloning Stem Cells 2004;6:386-391.

10 Kavalier F: Investigation of recurrent miscarriages. BMJ 2005;331:121-122.

11 Goldman BD: The circadian timing system and reproduction in mammals. Steroids 1999;64:679-685.

12 Turek FW: Biological rhythms in reproductive processes. Horm Res 1992;37:93-98.

13 Kovanen L, Saarikoski ST, Aromaa A, Lonnqvist J, Partonen T: Arntl (bmal1) and npas2 gene variants contribute to fertility and seasonality. PLoS One 2010;5:e10007.

14 King DP, Zhao Y, Sangoram AM, Wilsbacher LD, Tanaka M, Antoch MP, Steeves TD, Vitaterna MH, Kornhauser JM, Lowrey PL, Turek FW, Takahashi JS: Positional cloning of the mouse circadian clock gene. Cell 1997;89:641-653.

15 Loh DH, Kuljis DA, Azuma L, Wu Y, Truong D, Wang HB, Colwell CS: Disrupted reproduction, estrous cycle, and circadian rhythms in female mice deficient in vasoactive intestinal peptide. J Biol Rhythms 2014;29:355-369.

16 Gaddameedhi S, Selby CP, Kemp MG, Ye R, Sancar A: The circadian clock controls sunburn apoptosis and erythema in mouse skin. J Invest Dermatol 2015;135:1119-1127.

17 Antoch MP, Gorbacheva VY, Vykhovanets O, Toshkov IA, Kondratov RV, Kondratova AA, Lee C, Nikitin AY: Disruption of the circadian clock due to the clock mutation has discrete effects on aging and carcinogenesis. Cell Cycle 2008;7:1197-1204.

18 Peyric E, Moore HA, Whitmore D: Circadian clock regulation of the cell cycle in the zebrafish intestine. PLoS One 2013;8:e73209.

19 Seo BN, Ryu JM, Yun SP, Jeon JH, Park SS, Oh KB, Park JK, Han HJ: Delphinidin prevents hypoxia-induced mouse embryonic stem cell apoptosis through reduction of intracellular reactive oxygen species-mediated activation of jnk and NF-kappaB, and akt inhibition. Apoptosis 2013;18:811-824.

20 Nolan GP, Ghosh S, Liou HC, Tempst P, Baltimore D: DNA binding and i kappa b inhibition of the cloned p65 subunit of NF-kappaB, a rel-related polypeptide. Cell 1991;64:961-969.

21 Chen LF, Greene WC: Shaping the nuclear action of NF-kappaB. Nat Rev Mol Cell Biol 2004;5:392-401.

22 Moon SK, Jung SY, Choi YH, Lee YC, Patterson C, Kim CH: Pdtc, metal chelating compound, induces g1 phase cell cycle arrest in vascular smooth muscle cells through inducing p21cip1 expression: Involvement of p38 mitogen activated protein kinase. J Cell Physiol 2004;198:310-323.

23 Liu SF, Ye X, Malik AB: Inhibition of NF-kappaB activation by pyrrolidine dithiocarbamate prevents in vivo expression of proinflammatory genes. Circulation 1999;100:1330-1337. 